\title{
Ciclos de mejora docente integrados en Anatomía Patológica: ¿primer paso hacia una enseñanza integrada?
}

\author{
ENRIQUe de ÁlaVA CASADO \\ Universidad de Sevilla \\ Departamento de Citología e Histología Normal \\ y Patológica y UGC Anatomía Patológica \\ Hospital Universitario Virgen del \\ Rocio-IBiS / AGS Osuna \\ ealava-ibis@us.es \\ http://dx.doi.org/10.12795/JDU.2018.i01.67 \\ Pp.: 1191-1209
}

\section{Breve resumen y palabras clave}

Nuestra hipótesis es que el aprendizaje de los alumnos mejoraría si se integrasen contenidos de diversas asignaturas y se adelantasen algunos contenidos clínicos al comienzo del Grado de Medicina. Para llevar a cabo este proyecto hemos empleado la metodología de ciclos de mejora docente, realizados en el Programa de Formación e Innovación Docente del Profesorado (FIDOP), pero la hemos empezado a aplicar al diseño de Ciclos de Mejora Docente Integrados (CMDI), que abarquen aspectos concretos de varias asignaturas. La evaluación de la actividad, según la técnica de escaleras de aprendizaje y evaluación, opiniones de los alumnos, y autoevaluación, ofrece algunos puntos de 
mejora, pero sugiere que la intervención ha sido acertada en líneas generales.

Palabras clave: asignatura, titulación, docencia universitaria, experimentación docente universitaria, Anatomía Patológica

\section{Contexto}

La asignatura donde se han realizado los ciclos de CMDI es "Anatomía Patológica", correspondiente al primer semestre del tercer curso del grado de Medicina. Consta de 9 créditos repartidos en cuatro clases teóricas más 3 horas de prácticas o seminarios semanales de una hora impartidas en el primer cuatrimestre del curso. Es una asignatura troncal y de formación básica (obligatoria). Los alumnos ya han cursado Histología y Anatomía. Consta de 50 temas, agrupados en 8 secciones temáticas. Imparto docencia en dos grupos de 100 y 70 alumnos, respectivamente. Asisten a clase habitualmente un $80 \%$ de los alumnos.

Conexión con el diseño previo: En el curso anterior planteé, dentro del Curso General de Docencia Universitaria dos ciclos de mejora, que correspondían a 8 temas / 8 horas docentes. En esta ocasión, incorporo un tercer ciclo de mejora, y alargo los dos ciclos previos. Uno de ellos es un ciclo de mejora integrado. En total los ciclos de mejora abarcarán 20 temas / 22 horas docentes. Las principales conclusiones que se desprenden del trabajo del curso pasado son:

- Los dos CMD del curso pasado correspondieron a la primera mitad del temario, en la que los alumnos aprenden los mecanismos generales de enfermedad (p.ej. neoplasias, alteraciones circulatorias). Sin embargo, la segunda mitad del temario, en el que se analizan enfermedades concretas órgano a órgano (p.ej. carcinoma de pulmón), no estaba representada en los CMD. 
- Hay contenidos muy nuevos en el área de las neoplasias que no están representados en el temario. Es por ejemplo el caso de la respuesta inmunitaria ante las neoplasias, así como la importancia del estroma conjuntivo de las mismas, cuyo interés es creciente por su implicación terapéutica. Curiosamente, el tejido conjuntivo se explica en Histología de 1o de medicina, aparentemente de manera descriptiva y sin conexión clínica. Este conocimiento fragmentado requiere una actuación de mejora.

- Es necesario adaptar los materiales de las clases correspondientes a los ciclos de mejora a los contenidos del mapa de contenidos para lograr una mayor coherencia.

\section{Diseño previo del Ciclo de Mejora Docente:}

Se diseñaron tres ciclos de mejora, que a partir de ahora denominaremos ciclo 1, 2 y 3. Debido a las limitaciones de espacio, resumimos drásticamente esta sección, que está en su versión completa en este enlace de Google Drive.

1. El ciclo nô1 corresponde al área temática de bases cardiovasculares de la enfermedad. Corresponde a los temas 7-9 del temario. Fue objeto del primer CMD del Curso Básico, pero ahora se consolida y completa con un sistema de evaluación completo.

2. El ciclo no 2 corresponde al área temática de las neoplasias, temas 17-21 del temario. Corresponde al segundo CMD del Curso Básico, pero tiene una innovación importante: dado que hay contenidos muy nuevos, relacionados con la inmunoterapia del cáncer y al valor del estroma neoplásico, lo hemos rediseñado en forma de ciclo de mejora integrado (CMI) con la asignatura de Histología en Primer Curso del Grado de Medicina. Nuestra hipótesis para este ciclo es que el aprendizaje de los alumnos mejoraría si 
se integrasen contenidos de diversas asignaturas y se adelantasen algunos contenidos clínicos al comienzo del Grado. Este diseño es la consecuencia de un proyecto de Apoyo a la Coordinación e Innovación Docente. Modalidad B - Redes de Colaboración para la Innovación Docente, Convocatoria 2018-19 del III Plan Propio de Docencia. La memoria y los materiales relacionados con la ejecución de este proyecto pueden consultarse en este enlace.

3. El ciclo no3 es un intento de explorar cómo mejorar la docencia de la segunda parte del programa, la dedicada a las características concretas de cada enfermedad. En este caso los contenidos dejan de ser conceptuales para ser, en su mayor parte procedimientos y datos. He elegido para ello una sección del programa (temas 32-36) dedicada a la patología digestiva y hepática, con casos clínicos sencillos y adaptados a los estudiantes de tercer curso de Medicina. Este ciclo aún no se ha realizado a fecha de cierre de esta comunicación.

\section{Principios didácticos argumentados comunes a los tres ciclos}

Respecto a los contenidos he hecho una reflexión sobre los contenidos más estructuradores (p.ej. concepto de neoplasia, cómo diagnosticar una neoplasia), los más conceptuales y los procedimentales. Además, se han incluido habilidades procedimentales como la de describir lesiones o la de hablar en público. También se han incluido valores, como el de la prevención o la investigación.

Respecto al modelo metodológico, éste se ha basado en el trabajo de casos clínicos de forma individual o grupal de manera previa o durante la clase, de forma que ayude a los estudiantes a ser capaces de relacionar los conceptos teóricos con la realidad empresarial. Este principio se ha basado en las recomendaciones de Bain (2007) y Finkel (2005) sobre el aprendizaje autónomo de los alumnos. 


\section{Modelo metodológico argumentado común para los tres ciclos}

En el modelo metodológico seguido en los ciclos de mejora, se alterna el debate sobre casos clínicos con las explicaciones teóricas que ayudan a la resolución de estos, tal y como se representa en la Figura 1.

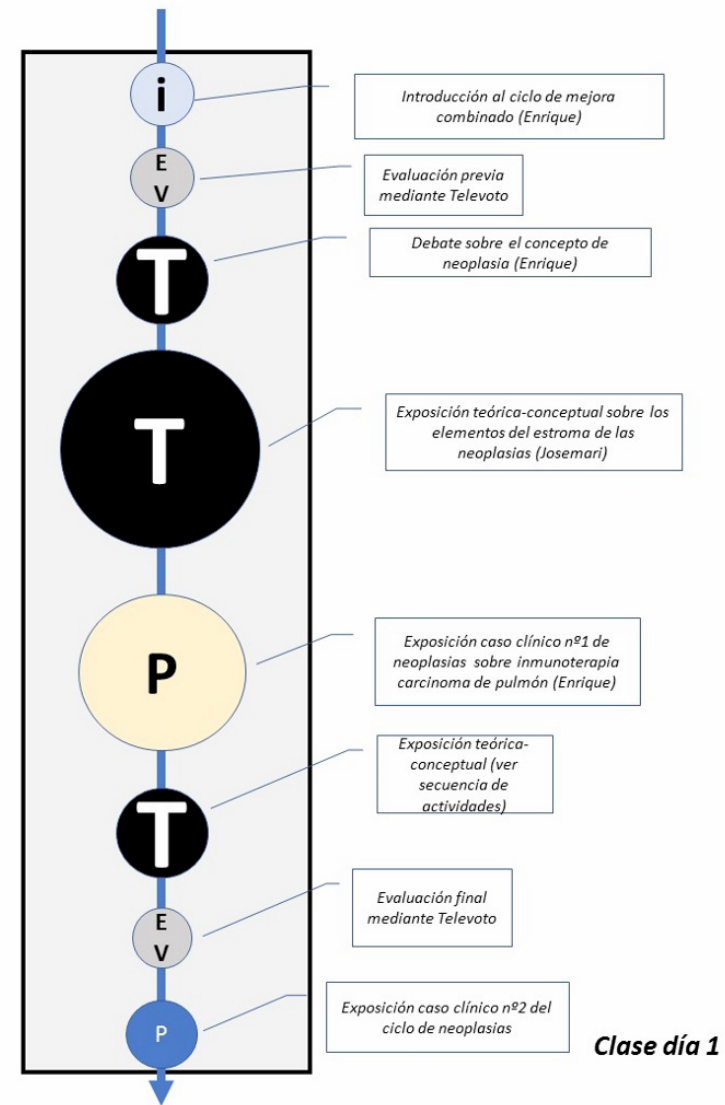

Figura 1: Modelo metodológico común a los tres ciclos de mejora Leyenda: P: Contenido práctico en relación con el caso clínico. AP: Actividad personal del alumno. I: introducción a la dinámica de clase. EV: Evaluación mediante televoto. T: Exposición teórica por parte del profesor de los contenidos teóricos en los que se apoya la resolución de las preguntas planteadas en el caso clínico

A continuación, detallo los aspectos específicos de cada ciclo de mejora:

Jornadas de Formación e Innovación Docente del Profesorado I № 1 (2018)

(c) Esta obra se distribuye con la licencia Creative Commons Reconocimiento-NoComercial-SinObraDerivada Internacional (CC BY-NC-ND 4.0.) 
Primer CMD

Mapa de contenidos y problemas o preguntas clave

Este primer ciclo de mejora se lleva a cabo en cuatro sesiones de 55 minutos en los que se imparten los temas referentes a las bases de la enfermedad relacionadas con la circulación de la sangre. Son temas importantes, aunque relativamente fáciles, y hacen por ello que la introducción del nuevo modelo metodológico por primera vez en el Grado no desconcierte, sino que estimule a los estudiantes.

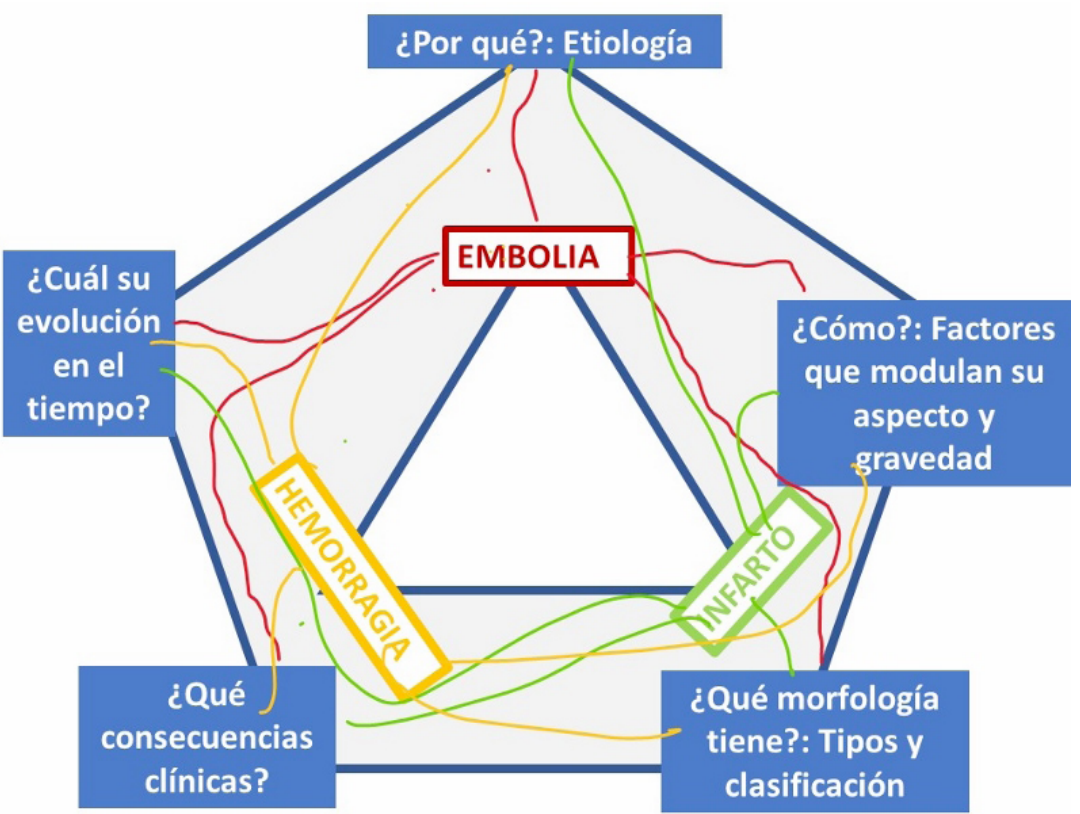

Figura 2. Mapa de contenidos de la sección de mecanismos de enfermedad circulatoria. Leyenda: El gráfico representa las cinco preguntas clave de las tres lesiones básicas de la enfermedad circulatoria (hemorragia, embolia, infarto). Las cinco preguntas clave están relacionadas entre sí (líneas gruesas), lo mismo que las tres lesiones clave. Las líneas finas de color representan las relaciones entre cada uno de estos contenidos.

Segundo CMD

Mapa de contenidos y problemas o preguntas-clave

Jornadas de Formación e Innovación Docente del Profesorado I № 1 (2018) Esta obra se distribuye con la licencia Creative Commons Reconocimiento-NoComercial-SinObraDerivada Internacional (CC BY-NC-ND 4.0.) 
El ciclo de mejora se realiza en seis sesiones de $55 \mathrm{mi}-$ nutos de clase, en las cuales se imparten los temas referentes a las neoplasias. Las neoplasias son un tema central en la Anatomía Patológica, y tanto los alumnos como el profesor están concienciados de su importancia dentro del temario. A continuación, muestro, por un lado, el mapa de contenidos general del CMD, y, a continuación, el mapa de la actuación integrada con Histología.

El mapa de contenidos general del CMD planteado se representa en la Figura 3

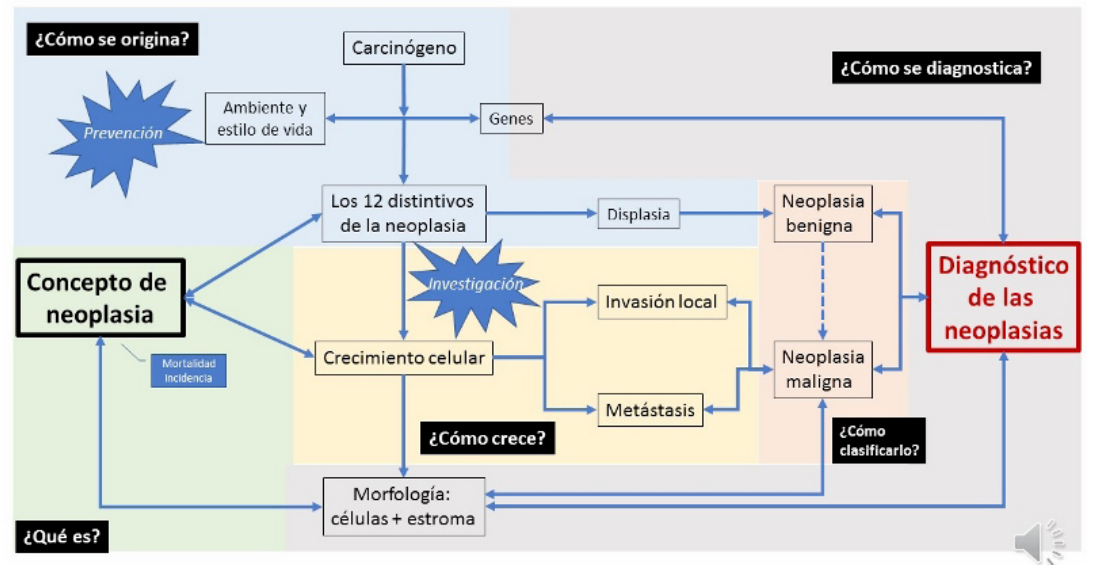

Figura 3: Mapa de contenidos general del segundo CMD

Leyenda: Conceptos fundamentales: negrita. Contenidos conceptuales: Negro; contenidos procedimentales: rojo. Estrellas: valores. Flechas: relaciones entre contenidos. Flecha discontinua: relación probable.

A continuación, se detallan las preguntas clave:

1. ¿Qué es o cómo se define una neoplasia?

1.bis ¿Cuáles son las características más importantes en la estructura normal del estroma conjuntivo de las neoplasias? ¿Qué aspectos de la estructura y la función del estroma influyen en la respuesta clínica del cáncer? 
2. ¿Cómo se origina una neoplasia?

3. ¿Cómo crece y se disemina una neoplasia?

4. ¿Cómo se clasifica o se nombra una neoplasia?

5. ¿Cómo se diagnostica una neoplasia?

Se incluye un enlace a un vídeo cargado en la plataforma Youtube, donde describo la construcción del mapa de contenidos y cómo se integran las preguntas clave en el esquema: https://youtu.be/4XpwC A-GWY

La pregunta clave no1bis, al ser la pregunta que define la parte integrada con Histología, tiene un mapa de contenidos independiente pero que debería verse como un detalle integrado en el mapa mostrado con anterioridad (concretamente dentro del contenido "Morfología: células+estroma" de la pregunta clave "Diagnóstico de la neoplasia")

Jornadas de Formación e Innovación Docente del Profesorado | № 1 (2018) Esta obra se distribuye con la licencia Creative Commons 


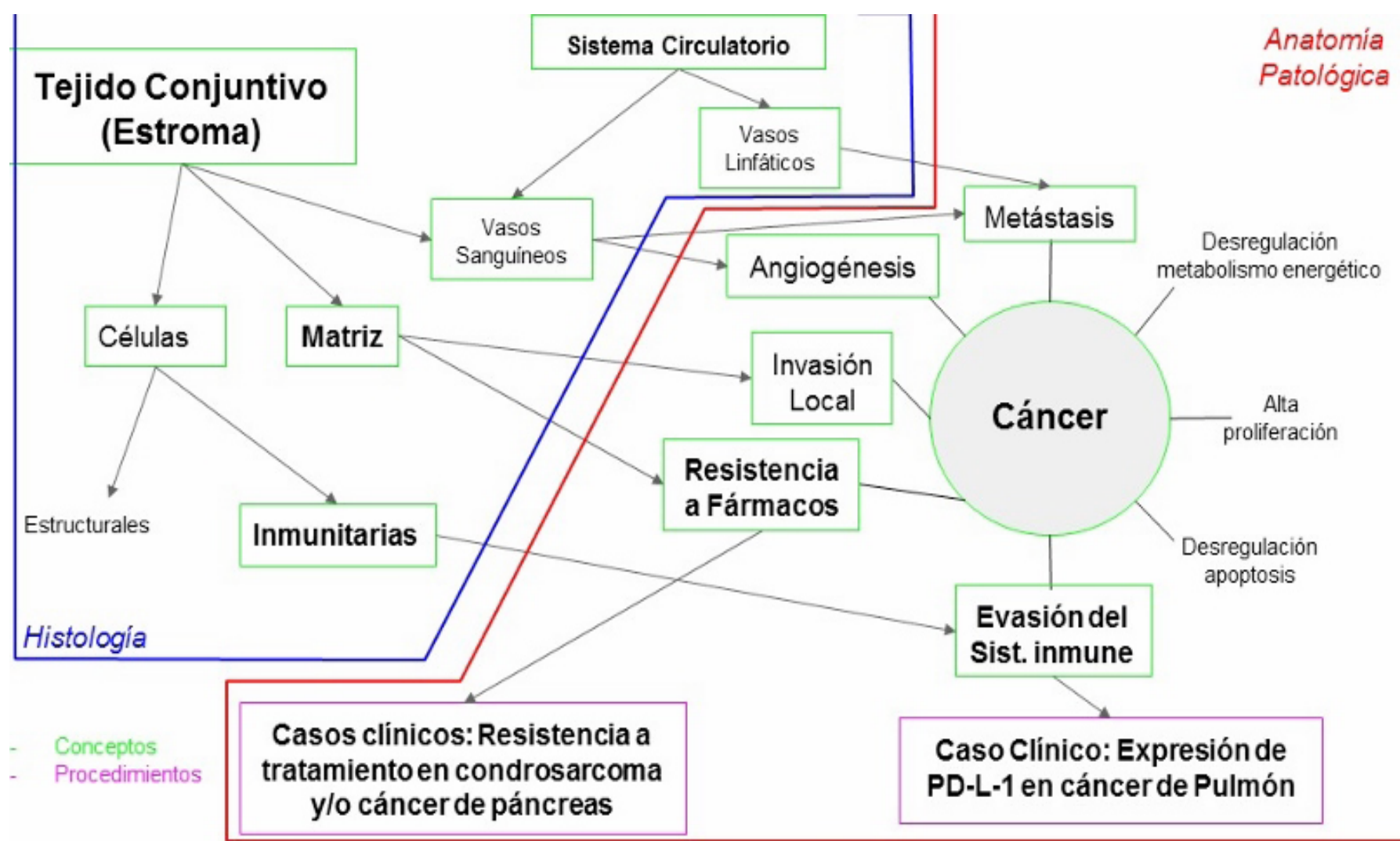

Figura 4. Mapa de contenidos de la parte integrada con Histología del CMD.

Jornadas de Formación e Innovación Docente del Profesorado | № 1 (2018)

(C) Esta obra se distribuye con la licencia Creative Commons 
Por razones de espacio omitimos el mapa de contenidos del CMDno3, los cuestionarios inicial/final y las secuencias de actividades que, no obstante, se pueden consultar en este enlace.

\section{Aplicación de los CMD}

\section{Ciclo de Mejora 1}

Resumen del diario de clase: El CMD 1 era relativamente breve. Un primer contacto con esta metodología para los alumnos. Durante la semana anterior al comienzo del CMD expliqué el cambio metodológico a los alumnos de manera sencilla, así como lo que esperaba de ellos. Les envié 5 días antes del comienzo un cuestionario de contenidos, que respondieron 18 personas (de 180 alumnos). El mismo cuestionario fue respondido por 11 alumnos después del CDM, y lo empleé para el diseño de escaleras de evaluación. La asistencia a clase fue del 90\% del alumnado y aproximadamente 40 alumnos participaron muy activamente de manera individual o grupal en las clases. Aunque estaba ya acostumbrado a los CMD del año anterior, en general me costó tener el control del tiempo según lo estipulado en la secuencia de actividades. Generalmente la discusión por grupos de los casos clínicos se prolongaba más de lo habitual gracias al animado ritmo de réplicas y contrarréplicas entre los estudiantes, con lo que al final me quedaba poco tiempo para hacer la exposición más formal. Sin embargo, esto no fue un gran problema porque esa exposición formal era breve en número y complejidad de los contenidos. Me di cuenta de que invitar a los alumnos a que prepararan por grupos los casos clínicos de la clase siguiente era algo muy exigente para ellos y que sus explicaciones en clase resultaban un poco confusas para un buen sector de alumnos, con lo que para los últimos días del ciclo mantuve la secuencia de actividades, pero cambiando la exposición grupal por sorteo por una preparación y exposición individual voluntaria de los 
alumnos. Les pedí que evaluaran específicamente este aspecto. Al final del ciclo les pasé un cuestionario de satisfacción que rellenaron 19 personas (curiosamente, más de las que respondieron por segunda vez al cuestionario de contenidos, 11). El promedio de calificación que me otorgaron fue de 8,22. Destaco la buena aceptación de la metodología por la mayoría; los aspectos más contestados fueron el elevado grado de exigencia, y el que al no ser una clase formal algunos alumnos se sentían inseguros. Varios alumnos me pidieron que, en lugar de empezar el tema con el caso práctico, podría empezarlo con la teoría, para poder aprovechar mejor la experiencia de búsqueda de información, mientras que otros pidieron que fuera muy claro en las conclusiones de cada tema. Otros pidieron que no hubiera caso clínico todos los días para poderse centrar también en otras asignaturas.

\section{Evaluación mediante escaleras de aprendizaje:}

Los cuestionarios de contenidos tenían 5 preguntas. He construido escaleras correspondientes a las 5 preguntas según la metodología descrita en las referencias bibliográficas de esta comunicación (Porlán R, 2017). Sin embargo, por razones de espacio, voy a mostrar las dos que considero más representativas. En una de ellas por el notable progreso en el aprendizaje, y en otra porque éste no ha sido tan satisfactorio.

3. ¿Qué morfología pueden tener las lesiones derivadas de las tres alteraciones hemodinámicas?

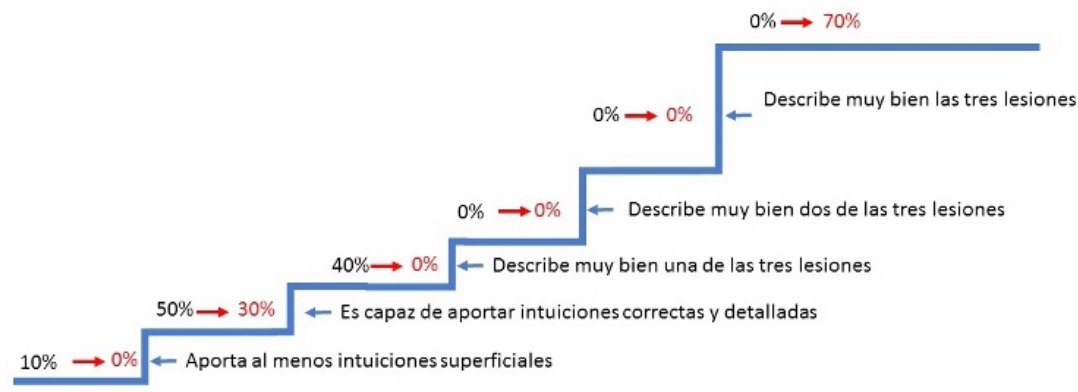

Figura 5. Escaleras de aprendizaje relacionadas con la pregunta guía no3 del CMDno1: ¿Cómo es la morfología de las lesiones? 
En la respuesta a esta pregunta se observa un notable avance en el aprendizaje de cuáles son las lesiones asociadas a la trombosis, hemorragia y embolia. Aunque muchos alumnos tenían intuiciones más o menos precisas acerca de dichas lesiones, aproximadamente el 70\% de ellos fueron capaces al final del ciclo de realizar una descripción satisfactoria de las lesiones.

Como conclusión para la pregunta guía 3 del CMDnô1, creo que hay que mantener la metodología docente ligada a este aspecto para el próximo curso

\section{4. ¿Qué consecuencias clínicas tienen estas alteraciones?}

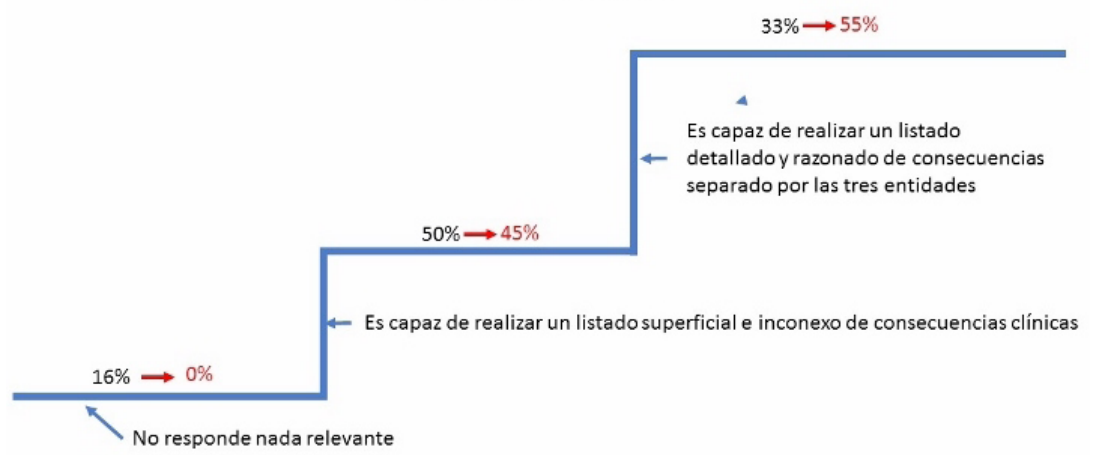

Figura 6. Escaleras de aprendizaje relacionadas con la pregunta guía no4 del CMDno1: ¿Qué consecuencias clínicas tienen estas alteraciones?

En la respuesta a esta pregunta, por el contrario, no se apreció ningún progreso significativo. Es verdad que el número de alumnos que no fue capaz de responder de manera coherente a esta pregunta se redujo al $0 \%$ tras el ciclo de mejora, pero, por el contrario, no hemos conseguido que la mayoría de los alumnos vayan más allá de un listado superficial e inconexo de consecuencias clínicas, no individualizadas para cada una de las tres entidades principales. Es posible que esto se deba a que haya dejado una longitud máxima no demasiado grande para la respuesta a esta pregunta y a que no haya pedido explícitamente en su enunciado una lista coherente y pormenorizada para cada 
tipo de entidad, pero había dado por supuesto que un conocimiento maduro ayuda a relacionar cada lesión con sus consecuencias clínicas.

Como conclusión para la pregunta guía no4 del CMDn우, creo que para el próximo año fomentaré especialmente la adquisición de contenidos procedimentales que les permitan asociar a cada lesión su consecuencia clínica a través de casos clínicos.

\section{Ciclo de mejora 2}

Resumen del diario de clase:

Viernes 19 de octubre

Este día realizamos la parte integrada del ciclo. Acude José María Fernández Santos. La primera clase explico a los alumnos de nuevo cómo va a ser el ciclo. Comienzo dialogando con los alumnos acerca del concepto de neoplasia, respondiendo la primera pregunta. El diálogo es bueno en el grupo b, a pesar de que faltan unos 20 alumnos para llenar la clase. Sin embargo, en el grupo d, la clase está llena hasta arriba, y los alumnos participan con mucho ánimo y un extenso diálogo. En la segunda parte de la clase el profesor José María Fernández Santos comenta su visión del estroma y algunas aplicaciones. En mi opinión, en la primera de las dos clases la explicación de José María es demasiado larga y me obliga a correr a mí en la tercera parte. Sin embargo, en la segunda clase el equilibrio entre las partes es mayor, y me deja más tiempo para explicar la tercera parte. Mientras cuento el caso clínico de la inmunoterapia veo como he captado la atención de los alumnos; creo que salen con la impresión de haber aprendido algo importante. Despiden la clase con un aplauso.

Lunes 22. Dedico la clase a responder a la pregunta de cómo el ambiente y los hábitos ayudan a que se desarrolle el cáncer. La clase se graba en uno de los dos grupos. Tengo la impresión de que invierto demasiado tiempo 
en la introducción teórica de la epidemiología y no consigo arrancar un diálogo claro con los alumnos al principio, hasta que no abordamos las 10 recomendaciones de Europa contra el cáncer. Tengo en los dos grupos la sensación de haber hablado demasiado.

Martes 23. Las dos clases mucho más dialogadas y centradas en las preguntas relevantes. El caso clínico está muy bien elegido porque ejemplifica las relaciones entre los conceptos de localización subcelular, tipo de protooncogén.

Miércoles 24. Muy participativos. Los alumnos se lían un poco con la explicación que relaciona el caso clínico donde hablo de la afectación de márgenes quirúrgicos con la actuación que debe llevar un cirujano cuando lee un informe de Anatomía Patológica con bordes libres/afectos. Esa intuición se me confirma porque les pregunto en el examen del 5 de noviembre una pregunta muy relacionada y pocos alumnos la aciertan. En general creo que los alumnos estaban previamente expuestos a contenidos sobre bases moleculares del cáncer

Jueves 25. Muy participativos. Explicamos cómo se diagnostica el cáncer, 'echando toda la carne en el asador', explicando de manera emotiva y experiencial cómo es la vida de un patólogo en un hospital (hay que intentar hacer adeptos ya en tercer curso de Medicina). Siento que los alumnos se sienten identificados con los procedimientos asociados al diagnóstico de las neoplasias y disfrutan de la clase.

En general noto que dedico bastante menos tiempo al caso clínico inicial y a su debate, y tengo más diapositivas de powerpoint que en el ciclo nô1, pero noto que los alumnos valoran mejor este equilibrio de tiempos, quizás más cercano a la clase tradicional que lo que he realizado en el CMDno1, que fue más radical. 
Al final del ciclo les pasé un cuestionario de satisfacción que rellenaron 10 personas. El promedio de calificación que me otorgaron fue de 8,66. Todos ellos manifestaron que habían aprendido más en el CMDno2 que en el CMDnㅇ․ Han valorado bien que los casos clínicos se trabajen de manera individual y que las clases sean un poco más dirigidas. Varios de ellos me manifiestan que prefieren que los 'deberes' que les envie en forma de casos clínicos sean después de impartir los contenidos principales en clase y no antes.

Evaluación mediante escaleras de aprendizaje:

Los cuestionarios de contenidos tenían 6 preguntas, 5 de ellas comunes con el CMD del año 2017. He construido escaleras correspondientes a las 6 preguntas según la metodología descrita en las referencias bibliográficas de esta comunicación (Porlán R, 2017). Sin embargo, por razones de espacio, voy a mostrar las dos que considero más representativas. Una de ellas porque es nueva (no estaba en el CMD de 2017 y corresponde a la parte integrada del CMD; la he denominado 1bis, para poder seguir comparando con facilidad las preguntas de 2018 con las homónimas de 2017) y la otra, porque supone un progreso respecto a la misma escalera de 2017.

CMDno2. Pregunta 1 bis. ¿Estroma y respuesta clínica al tratamiento del cáncer?

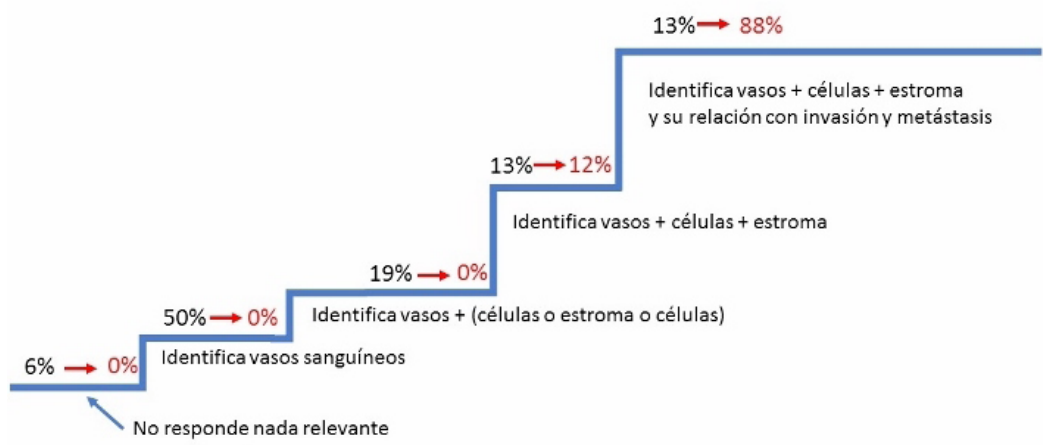

Figura 7. Escalera correspondiente a la pregunta guía 1bis del CMDnㅇ2

Jornadas de Formación e Innovación Docente del Profesorado | № 1 (2018) Esta obra se distribuye con la licencia Creative Commons 
Me parece un avance significativo en un área en pleno desarrollo en la medicina actual. Creo que la comprensión del papel del estroma de la neoplasia es un contenido muy transversal a toda la biología y la clínica de los pacientes con neoplasias malignas y abre la puerta de nuevos tratamientos: los alumnos han comprendido esto de manera excelente. Como conclusión, mantendremos y extenderemos la integración en los CMD del próximo curso.

CMDn-2. Pregunta 5. ¿Cómo se diagnostica una neoplasia? 2017

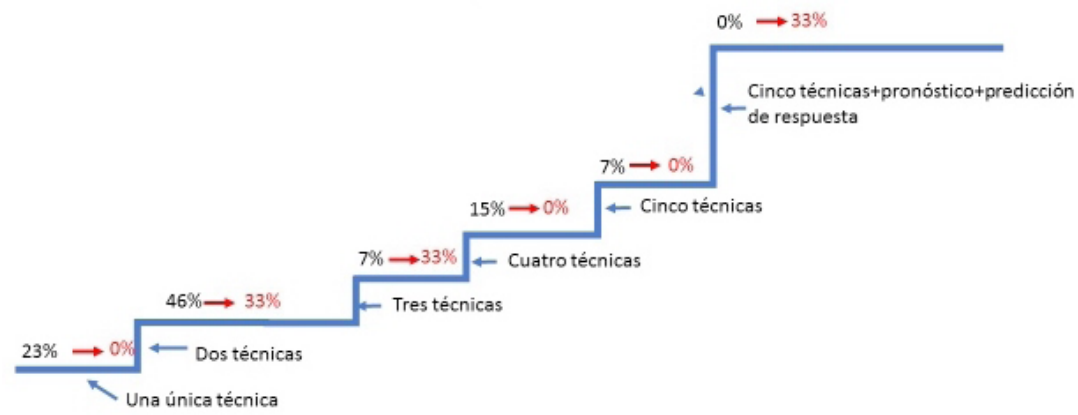

Figura 8. Escalera correspondiente a la pregunta guía no5 del CMDno2 (¿Cómo se diagnostican las neoplasias?), comparando los resultados de 2018 (arriba) con los de 2017 (abajo).

La comparación de las escaleras de aprendizaje entre los dos años arroja resultados mixtos. Por un lado, se cubre en buena parte una carencia de los estudiantes de 2017, que no comprendían toda la complejidad y el número de técnicas necesarias para llegar a un diagnóstico de las neoplasias; mientras que el $66 \%$ de los estudiantes del año pasado no era capaz de nombrar más de tres tipos de técnicas diagnósticas, ese porcentaje baja en 2018 al $12 \%$. Sin embargo, por otro lado, desciende el porcentaje de estudiantes que asocian el uso de biomarcadores predictivos al diagnóstico desde el 33\% de 2017 al 12\% de 2018. Como conclusión, mantendremos los aspectos de la docencia de este año ligados a la tecnología y a la multidisciplinariedad diagnóstica, pero reforzaremos (todavía en lo 
que queda de este curso) el papel de los biomarcadores predictivos de respuesta al tratamiento como parte indispensable para el diagnóstico de las neoplasias.

\section{Evaluación del CMD. Conclusiones}

Una vez concluido el ciclo de mejora destacamos los avances más significativos:

\section{Avances conseguidos:}

- Los alumnos han captado muy bien los contenidos clave de los temas ligados a los CMDs. Aunque las encuestas Socrative de los días de clase ya daban valores elevados de aciertos, la corrección del examen del día 5 de noviembre ha revelado calificaciones mucho más elevadas que las del examen análogo del curso anterior (siendo de complejidad similar).

- Estoy particularmente satisfecho del diseño y ejecución de una parte integrada del CMDno2. Creo que es un camino claro para seguir profundizando en la mejora docente.

- Los alumnos se están acostumbrando y disfrutan de la docencia centrada en casos clínicos. Claramente les ayuda a integrar la visión clínica en su aprendizaje de la Anatomía Patológica. Se sienten más médicos; hay que tener en cuenta que son alumnos de tercero en un Grado de 6 años, y que es la primera asignatura abiertamente médica que reciben. Lo expresan así en una encuesta de satisfacción que les he realizado tras la finalización del ciclo y del examen (sin conocer las calificaciones de este).

- La participación de los alumnos en la clase, siempre elevada, es ahora más extensa y ordenada.

- He sido capaz de incluir con cierta flexibilidad y creatividad algunas mejoras metodológicas sobre la 
marcha (debates acerca de ciertos conceptos o valores), que han sido útiles para el aprendizaje, aunque hayan consumido mucho tiempo.

\section{Dificultades encontradas:}

- Un aspecto que ha condicionado bastante este CMD, en particular la parte integrada, es que los tres profesores implicados impartimos docencia en tres campus diferentes y a las mismas horas. Esto nos ha obligado a notables ajustes de agenda para poder realizarlo.

\section{Aspectos a incluir:}

- Mantener la metodología, quizás menos radical que en el CMDno1, y más parecida al CMDno2. De hecho, ahora que estoy acabando de planificar el CMDno3, me doy cuenta de que ya no hay diferencias entre los CMD y las clases 'normales' en las que ya inconscientemente incluyo el mismo modelo metodológico que en el CMD

- Progresar en la integración en el 2019-2020, pero ahora por el lado clínico, sobre todo con los profesores de patología médica y quirúrgica de 40 y 5o curso

- Dedicar más tiempo a los sistemas de clasificación de las neoplasias.

- Explorar la posibilidad de incluir casos clínicos después de haber impartido algunos contenidos conceptuales y no antes porque, especialmente a principio del curso, puede liar más a un 30\% de alumnos que beneficiarles. Quizás esto sea menos problemático según avanza el curso y su cambio de rol deba ser gradual.

- Reducir y priorizar el no de diapositivas en algunas clases sobre neoplasias, donde el caso clínico dejaba poco tiempo para poder explicar diapositivas en modo 'magistral'. 


\section{Bibliografía}

Blain, K. (2007). Lo que hacen los mejores profesores de Universidad. Valencia: Publicaciones de la Universitat de Valéncia.

Finkel, D. (2008). Dar clase con la boca cerrada. Valencia: Publicaciones de la Universitat de Valéncia.

Porlán R (Coord.).(2017). Enseñanza Universitaria, cómo mejorarla. Madrid. Ediciones Morata. 